CLINICAL STUDY

\title{
Dimethylarginines: their vascular and metabolic roles in Africans and Caucasians
}

Aletta E Schutte, Rudolph Schutte, Hugo W Huisman, Johannes M van Rooyen, Carla M T Fourie, Leoné Malan, Nico T Malan, Edzard Schwedhelm ${ }^{1}$, Sebastian Strimbeanu ${ }^{1}$, Maike Anderssohn ${ }^{1}$ and Rainer H Böger ${ }^{1}$

Hypertension in Africa Research Team (HART), School for Physiology, Nutrition and Consumer Sciences, North-West University (Potchefstroom Campus), Private Bag X6001, Potchefstroom 2520, South Africa and ${ }^{1}$ Clinical Pharmacology Unit, Institute of Experimental and Clinical Pharmacology and Toxicology, University Medical Center Hamburg-Eppendorf, 20246 Hamburg, Germany

(Correspondence should be addressed to A E Schutte; Email: alta.schutte@nwu.ac.za)

\begin{abstract}
Objective: Alarming increases in hypertension and type 2 diabetes among Africans accentuate the need to identify factors that could serve as targets for prevention or treatment. In Caucasian populations, asymmetric dimethylarginine (ADMA), the predominant endogenous nitric oxide synthase inhibitor, is associated with cardiovascular disease and insulin resistance (IR). ADMA's counterpart, symmetric dimethylarginine (SDMA), originally thought to be inert, was recently also linked with cardiovascular risk. Since little information regarding ADMA or SDMA is available for Africans, our aim was to explore the relationships of ADMA and SDMA with measures of arterial stiffness and IR in Africans and Caucasians from South Africa.

Methods: The study consisted of 235 nonsmoking, nondiabetic, nonobese, human immunodeficiency virus-uninfected Africans $(n=64)$ and Caucasians $(n=171)$, aged 20-70 years. We measured blood pressure, pulse wave velocity, ADMA, SDMA, and IR (homeostasis model assessment, HOMA).

Results: African and Caucasian men had similar ADMA and SDMA, whereas Caucasian women had higher ADMA and SDMA than African women $(P<0.05)$. African men and Caucasian women indicated strong correlations of ADMA with arterial stiffness $(r=0.47, P=0.021 ; r=0.26, P=0.008)$, confirmed in multivariate analyses. Caucasian participants showed negative associations between SDMA and HOMA, being strongest in the men $(r=-0.41 ; P=0.002)$.

Conclusion: Our results indicate that ADMA is independently associated with vascular dysfunction in African men and Caucasian women. A strong, independent negative association of SDMA with IR was found only in Caucasian participants. The molecular explanation for this is unclear, but these findings motivate experimental studies that could shed more light on these relationships.
\end{abstract}

European Journal of Endocrinology 162 525-533

\section{Introduction}

Extensive epidemiological studies show that hypertension is one of the most common cardiovascular ailments in sub-Saharan Africa (1-4). Furthermore, the increasing incidence of diabetes (5) in Africa will aggravate the severity of renal and cardiac damage caused by any given blood pressure (BP) level (1). Although a high prevalence of known modifiable risk factors for atherosclerotic disease and hypertensive heart disease is detected in black South African individuals (6), these conventional risk factors do not account for all of their increased risk (7). It is therefore imperative to identify additional markers that might explain their increased risk and may contribute to the early detection and prevention of vascular and metabolic abnormalities.

In Caucasian populations, elevated concentrations of asymmetric dimethylarginine (ADMA) have consistently been associated with cardiovascular disease
(8-12) and insulin resistance (IR) (13-15). ADMA, which is regarded as the predominant endogenous nitric oxide (NO) synthase inhibitor (9), is found in all human tissues and biological fluids, and is generated by the post-translational methylation of arginine residues in proteins (8). Owing to its inhibitory effect on NO synthase, ADMA has been implicated in the impairment of NO generation in a variety of cardiovascular diseases (16). Melikian et al. (7) have indicated that ADMA levels were significantly higher in Africans compared to Europeans and they suggested that this may contribute to higher incidence of cardiovascular disease in black Africans. ADMA therefore seems to be a suitable candidate for the early detection of cardiovascular and metabolic disease in Africans.

Unlike ADMA, its counterpart, symmetric dimethylarginine (SDMA), does not inhibit NO synthase (17). Thus, this structural isomer was thought to be inert (18) and it has not been evaluated in most studies 
focusing on cardiovascular disease (8). However, recent publications suggest that SDMA has a similar predictive value for cardiovascular risk than ADMA (8). We also reported recently that SDMA is a prospective marker of total mortality after ischemic stroke (19). Several explanations for this observation including increased formation of reactive oxygen species, competition for L-arginine transport into endothelial cells, or valid reflection of renal function by SDMA have been proposed $(18,20,21)$.

In addition to SDMA's possible vascular role, Zsuga et al. (22) also suggest that the ADMA/SDMA ratio is a significant determinant of IR. Very little is known regarding the possible role of SDMA with regards to IR or with regards to its concentration or functions. To the best knowledge of the authors, no literature is available regarding SDMA in black populations. Nonetheless, the available evidence supports the hypothesis that SDMA may not be as inert as initially thought.

Current knowledge on these two dimethylarginines mainly focused on Caucasian populations, with only few studies investigating black populations in the USA and Europe who may (or may not) be genetically and environmentally different from black South Africans (1). The aim of this study was therefore to explore and evaluate the associations of ADMA and SDMA with measures of arterial stiffness and IR in African and Caucasian people from South Africa.

\section{Methods}

\section{Study design and subject selection}

The SAfrEIC study (South African study regarding the influence of Sex, Age and Ethnicity on Insulin sensitivity and Cardiovascular function) was a cross-sectional study that originally involved 750 African and Caucasian men and women volunteers from urban areas of the North-West Province of South Africa (aged 20-70 years). This availability sample excluded participants that were apparently ill, pregnant, or lactating. For the purposes of the present study, a sub-sample of 64 African and 171 Caucasian participants were selected based on the following exclusion criteria: smokers $(n=218)$; nonfasting $(n=11)$; obese (i.e. body mass index $\left.(\mathrm{BMI}) \geq 30 \mathrm{~kg} / \mathrm{m}^{2}\right)(n=84)$; use of antihypertensive-, antidiabetic-, anti-inflammatory-, or lipid-lowering medication $(n=86)$; missing data $(n=1)$; previous diagnosis of any serious chronic illness, e.g. diabetes $(n=2)$; or human immunodeficiency viral (HIV) infection $(n=115)$ (except hypertension). The main reason for the discrepancy in the final sample sizes of the ethnic groups is that $33 \%$ of the African participants were HIV infected $(n=114)$. To provide $80 \%$ power at $5 \%$ significance and taking a difference of $0.10 \mu \mathrm{mol} / \mathrm{l}$ in ADMA as the main outcome variable as significant, a total number of 15 subjects were needed per group.
The Ethics Committee of the North-West University (Potchefstroom campus) approved this study and all participants gave written informed consent after the procedures were thoroughly explained to them. African field workers were available to convey the information to the African participants in their home language.

\section{Organisational procedures}

The participants were invited for a nonrecurring clinical examination to a Metabolic Unit facility on the campus of the North-West University, South Africa. They arrived at $0700 \mathrm{~h}$, were introduced to the setup and, after the organisational procedures had been explained to them (with the assistance of the African field workers), they all signed informed consent forms. During the course of the morning, basic health and demographic questionnaires were completed. A fasting blood sample was collected by a registered nurse from the brachial vein branches using sterile winged infusion sets and syringes, and anthropometric measurements were taken in a private bedroom. Afterwards, cardiovascular measurements were made. On completion of the measurements, each participant received breakfast as well as a small financial compensation for travel expenses. In the event of a subject being identified with any abnormalities (such as hypertension or diabetes), the participant was referred to their local clinic, hospital, or physician. Each participant received a report containing their basic health information.

\section{Biochemical analyses}

The participants were required to fast overnight before the fasting blood glucose level was determined. Capillary blood glucose was directly measured in the Metabolic Unit by a nurse by means of an enzymatic method to screen for diabetes mellitus (LifeScan SureStep Blood Glucose Monitoring System, LifeScan Inc., Milputas, CA 9535, USA). Serum and plasma samples were also taken and stored at $-80{ }^{\circ} \mathrm{C}$ until analysis. Serum blood glucose, the lipogram, creatinine, and $\gamma$-glutamyltransferase were determined later in the laboratory with the Konelab auto-analyzer (Thermo Fisher Scientific Oy, Vantaa, Finland). Creatinine clearance was estimated using the Cockcroft-Gault method (23). Insulin was determined by making use of the ST AIA-PACK IRI kit (Cat. No 025260) using two-site immunoenzymometric assay. IR was estimated by the homeostasis model assessment-IR (HOMA-IR) index, calculated as the product of fasting glucose and insulin divided by 22.5. HIV status was determined immediately after blood sampling with a rapid test according to the protocol of the National Department of Health of South Africa. Serum was used for testing with the First Response test (PMC Medical, Daman, India) and was repeated with Pareeshak test (BHAT Bio-tech, Bangalore, India) for confirmation. 
Mass spectrometric determination of ADMA and SDMA was performed as described elsewhere by using a fully validated high throughput liquid chromatography-tandem mass spectrometry (MS)/MS assay, which is commercially available (DLD Diagnostika, Hamburg, Germany) (24, 25).

\section{Anthropometric measurements}

Body height, weight, and waist and hip circumferences of each participant were taken according to standard procedures (26). The circumferences were measured in triplicate with a flexible metallic measuring tape (Holtain unstretchable metal tape). Maximum height was measured to the nearest $0.1 \mathrm{~cm}$ using the Invicta Stadiometer (IP 1465, Leicester, UK). Weight was measured to the nearest $0.1 \mathrm{~kg}$ using a digital scale (Precision Health Scale, A \& D Company, Tokyo, Japan).

\section{Cardiovascular measurements}

After a 10 min rest in the sitting position, BP (systolic and diastolic) and heart rate were measured using the OMRON HEM-757 apparatus, with the BP cuff on the left upper arm. Two measurements were taken, with a 5 min rest interval.
Pulse wave velocity (PWV) was measured using noninvasively accessible superficial pulses and the Complior SP device (Artech Medical, Pantin, France) in an elastic-muscular mixed arterial segment over the carotid dorsalis pedis segment. All measurements were taken by the same two observers for all participants. The PWV was measured on the left side of each participant, while the subject was in a supine position.

\section{Statistical analyses}

All statistical analyses were done using Statistica version 8 (StatSoft, Inc., Tulsa, OK, USA) software. Variables with a non-Gaussian distribution (namely waist circumference, BP, $\gamma$-glutamyltransferase, triglycerides (TG), glucose, insulin, and HOMA) were logarithmically transformed, and the central tendency and spread represented by the geometric mean and the 5th and 95th percentile intervals. Independent samples $t$-tests were performed to compare the two ethnic groups. We performed partial correlations of ADMA or SDMA and variables associated with arterial function and IR while adjusting for confounders as indicated in Table 1. Fisher's Z-transformation was performed to determine $95 \%$ confidence intervals

Table 1 Partial correlations of asymmetric dimethylarginine (ADMA) and symmetric dimethylarginine (SDMA) with markers of vascular function and insulin resistance $(95 \%$ confidence intervals of $r)$.

\begin{tabular}{|c|c|c|c|c|}
\hline & \multicolumn{2}{|c|}{ African men $(n=28)$} & \multicolumn{2}{|c|}{ Caucasian men $(n=66)$} \\
\hline & $\mathrm{ADMA}^{\mathrm{a}}$ & SDMA $^{b}$ & ADMA & SDMA \\
\hline Systolic blood pressure (log) & $\begin{array}{l}r=0.29(-0.12 ; 0.61) \\
P=0.16\end{array}$ & $\begin{array}{l}r=0.21(-0.22 ; 0.57) \\
P=0.34\end{array}$ & $\begin{array}{l}r=0.06(-0.19 ; 0.30) \\
P=0.65\end{array}$ & $\begin{array}{l}r=0.03(-0.22 ; 0.28) \\
P=0.83\end{array}$ \\
\hline Diastolic blood pressure (log) & $\begin{array}{l}r=0.05(-0.35 ; 0.44) \\
P=0.80\end{array}$ & $\begin{array}{l}r=0.11(-0.30 ; 0.48) \\
P=0.62\end{array}$ & $\begin{array}{l}r=0.01(-0.24 ; 0.26) \\
P=0.96\end{array}$ & $\begin{array}{l}r=-0.11(-0.35 ; 0.15) \\
P=0.44\end{array}$ \\
\hline Pulse wave velocity ${ }^{c}$ & $\begin{array}{l}r=0.47(0.08 ; 0.73) \\
P=0.021\end{array}$ & $\begin{array}{l}r=-0.06(-0.47 ; 0.37) \\
P=0.80\end{array}$ & $\begin{array}{l}r=-0.18(-0.41 ; 0.07) \\
P=0.16\end{array}$ & $\begin{array}{l}r=0.07(-0.19 ; 0.32) \\
P=0.59\end{array}$ \\
\hline Fasting insulin (log) & & $\begin{array}{l}r=0.05(-0.37 ; 0.45) \\
P=0.84\end{array}$ & $\begin{array}{l}r=-0.16(-0.39 ; 0.09) \\
P=0.23\end{array}$ & $\begin{array}{l}r=-0.41(-0.60 ;-0.18) \\
P=0.002\end{array}$ \\
\hline \multirow[t]{3}{*}{ HOMA (log) } & $\begin{array}{l}r=0.04(-0.36 ; 0.43) \\
P=0.86\end{array}$ & $\begin{array}{l}r=0.05(-0.37 ; 0.45) \\
P=0.83\end{array}$ & $\begin{array}{l}r=-0.16(-0.39 ; 0.09) \\
P=0.24\end{array}$ & $\begin{array}{l}r=-0.41(-0.60 ;-0.18) \\
P=0.002\end{array}$ \\
\hline & \multicolumn{2}{|c|}{ African women $(n=36)$} & \multicolumn{2}{|c|}{ Caucasian women $(n=105)$} \\
\hline & $\mathrm{ADMA}^{\mathrm{a}}$ & $\mathrm{SDMA}^{\mathrm{b}}$ & ADMA & SDMA \\
\hline Systolic blood pressure (log) & $\begin{array}{l}r=-0.30(-0.58 ; 0.05) \\
P=0.09\end{array}$ & $\begin{array}{l}r=-0.09(-0.43 ; 0.27) \\
P=0.64\end{array}$ & $\begin{array}{l}r=0.18(-0.02 ; 0.36) \\
P=0.08\end{array}$ & $\begin{array}{l}r=0.07(-0.13 ; 0.26) \\
P=0.50\end{array}$ \\
\hline Diastolic blood pressure (log) & $\begin{array}{l}r=-0.25(-0.55 ; 0.10) \\
P=0.17\end{array}$ & $\begin{array}{l}r=-0.10(-0.44 ; 0.26) \\
P=0.60\end{array}$ & $\begin{array}{l}r=0.15(-0.05 ; 0.33) \\
P=0.16\end{array}$ & $\begin{array}{l}r=0.08(-0.12 ; 0.27) \\
P=0.44\end{array}$ \\
\hline Pulse wave velocity & $\begin{array}{l}r=0.10(-0.26 ; 0.43) \\
P=0.57\end{array}$ & $\begin{array}{l}r=0.15(-0.22 ; 0.48) \\
P=0.43\end{array}$ & $\begin{array}{l}r=0.26(0.07 ; 0.43) \\
P=0.008\end{array}$ & $\begin{array}{l}r=0.22(0.02 ; 0.40) \\
P=0.032\end{array}$ \\
\hline Fasting insulin (log) & $\begin{array}{l}r=0.20(-0.15 ; 0.51) \\
P=0.27\end{array}$ & $\begin{array}{l}r=-0.02(-0.37 ; 0.34) \\
P=0.93\end{array}$ & $\begin{array}{l}r=0.01(-0.18 ; 0.20) \\
P=0.97\end{array}$ & $\begin{array}{l}r=-0.16(-0.35 ; 0.04) \\
P=0.13\end{array}$ \\
\hline HOMA (log) & $\begin{array}{l}r=0.20(-0.15 ; 0.51) \\
P=0.28\end{array}$ & $\begin{array}{l}r=0.01(-0.35 ; 0.36) \\
P=1.00\end{array}$ & $\begin{array}{l}r=0.01(-0.18 ; 0.20) \\
P=0.95\end{array}$ & $\begin{array}{l}r=-0.17(-0.35 ; 0.03) \\
P=0.11\end{array}$ \\
\hline
\end{tabular}

HOMA, homeostasis model assessment. Bold indicates statistical significance $(P<0.05)$.

${ }^{a}$ Adjusted for age, body mass index, and waist circumference.

${ }^{\mathrm{b}}$ Adjusted for age, body mass index, waist circumference, estimated creatinine clearance, and $\gamma$-glutamyltransferase.

${ }^{\mathrm{c} C}$ Correlations with pulse wave velocity were additionally adjusted for mean arterial pressure. 
of the partial correlations. Since alcohol intake is associated with higher ADMA levels (27), $\gamma$-glutamyltransferase was included in analyses being a marker of alcohol intake (28). We also found differences in $\gamma$-glutamyltransferase between the ethnic groups (Table 2). We plotted quartiles of HOMA $(\log )$ against SDMA while applying adjustments for known confounders namely age, BMI, waist circumference, $\gamma$-glutamyltransferase (28), and estimated creatinine clearance (21). We constructed multivariable linear regression models with forward stepwise selection for each gender and ethnic group for either ADMA or SDMA as dependent variable. Covariates included in the ADMA model were age, BMI, mean arterial pressure, PWV, TG, and $\gamma$-glutamyltransferase. The SDMA model was identical except for two additional covariates: HOMA (22) and estimated creatinine clearance (21). Similar additional multivariable regression models were analysed for each gender with either ADMA or SDMA as dependent variables, but we included ethnicity in the list of independent variables.

Table 2 Basic characteristics of African and Caucasian participants. Data are arithmetic mean \pm S.E.M. or geometric mean (5th and 95th percentile intervals) for logarithmically transformed variables.

\begin{tabular}{|c|c|c|c|}
\hline \multirow[b]{2}{*}{$n$} & \multirow{2}{*}{$\frac{\text { African men }}{28}$} & \multicolumn{2}{|l|}{ Caucasian men } \\
\hline & & 66 & $\boldsymbol{P}$ \\
\hline Age (years) & $34.9 \pm 14.0$ & $37.6 \pm 13.1$ & 0.39 \\
\hline \multicolumn{4}{|l|}{ Anthropometric measurements } \\
\hline Height $(\mathrm{m})$ & $1.70 \pm 0.05$ & $1.81 \pm 0.07$ & $<0.001$ \\
\hline Weight (kg) & $64.7 \pm 10.5$ & $83.6 \pm 10.9$ & $<0.001$ \\
\hline Body mass index $\left(\mathrm{kg} / \mathrm{m}^{2}\right)$ & $22.3 \pm 3.44$ & $25.4 \pm 2.51$ & $<0.001$ \\
\hline Waist circumference (cm) & $76.6(72.7 ; 80.6)$ & $86.4(84.3 ; 88.6)$ & $<0.001$ \\
\hline \multicolumn{4}{|l|}{ Cardiovascular measurements } \\
\hline Systolic blood pressure (mmHg) & $125(117 ; 132)$ & $120(118 ; 123)$ & 0.18 \\
\hline Diastolic blood pressure (mmHg) & $81.0(75.8 ; 86.5)$ & $76.5(74.8 ; 78.2)$ & 0.04 \\
\hline Pulse wave velocity $(\mathrm{m} / \mathrm{s})$ & $7.78 \pm 1.54$ & $7.91 \pm 1.24$ & 0.67 \\
\hline Estimated creatinine clearance $(\mathrm{ml} / \mathrm{min})$ & $126 \pm 29.8$ & $153 \pm 30.9$ & $<0.001$ \\
\hline$\gamma$-Glutamyltransferase (U/I) & $50.0(37.1 ; 67.5)$ & $32.2(28.5 ; 36.3)$ & 0.001 \\
\hline \multicolumn{4}{|l|}{ Lipid profile } \\
\hline HDL-cholesterol (mmol/l) & $1.39+0.45$ & $1.31+0.31$ & 0.31 \\
\hline Triglycerides $(\mathrm{mmol} / \mathrm{l})$ & $0.91(0.75 ; 1.09)$ & $1.09(0.96 ; 1.25)$ & 0.16 \\
\hline Fasting glucose (mmol/l) & $4.90(4.69 ; 5.11)$ & $5.41(5.24 ; 5.58)$ & $<0.001$ \\
\hline Fasting insulin $(\mu \mathrm{U} / \mathrm{ml})$ & $5.65(4.14 ; 7.72)$ & $6.25(5.33 ; 7.33)$ & 0.52 \\
\hline HOMA & $1.23(0.89 ; 1.71)$ & $1.52(1.28 ; 1.82)$ & 0.21 \\
\hline ADMA $(\mu \mathrm{mol} / \mathrm{l})$ & $0.60 \pm 0.12$ & $0.62 \pm 0.16$ & 0.62 \\
\hline \multirow[t]{2}{*}{ SDMA $(\mu \mathrm{mol} / \mathrm{l})$} & $0.54 \pm 0.11$ & $0.60 \pm 0.16$ & 0.08 \\
\hline & African women & Caucasian women & \\
\hline$n$ & 36 & 105 & $\boldsymbol{P}$ \\
\hline Age (years) & $37.8 \pm 12.3$ & $36.5 \pm 11.0$ & 0.54 \\
\hline \multicolumn{4}{|l|}{ Anthropometric measurements } \\
\hline Height (m) & $1.58 \pm 0.06$ & $1.66 \pm 0.05$ & $<0.001$ \\
\hline Weight (kg) & $59.7 \pm 10.1$ & $66.4 \pm 10.2$ & $<0.001$ \\
\hline Body mass index (kg/m²) & $23.7 \pm 3.67$ & $23.9 \pm 3.33$ & 0.77 \\
\hline Waist circumference $(\mathrm{cm})$ & $73.9(70.9 ; 77.1)$ & $74.3(72.8 ; 75.8)$ & 0.83 \\
\hline \multicolumn{4}{|l|}{ Cardiovascular measurements } \\
\hline Systolic blood pressure (mmHg) & $116(108 ; 124)$ & $109(107 ; 111)$ & 0.02 \\
\hline Diastolic blood pressure (mmHg) & $80.4(75.3 ; 85.9)$ & $72.4(70.7 ; 74.1)$ & $<0.001$ \\
\hline Pulse wave velocity $(\mathrm{m} / \mathrm{s})$ & $7.39 \pm 1.64$ & $7.42 \pm 1.01$ & 0.88 \\
\hline Estimated creatinine clearance $(\mathrm{ml} / \mathrm{min})$ & $96.2 \pm 25.3$ & $103 \pm 23.7$ & 0.16 \\
\hline$\gamma$-Glutamyltransferase $(\mathrm{U} / \mathrm{l})$ & $35.4(26.8 ; 46.8)$ & $21.3(19.5 ; 23.3)$ & $<0.001$ \\
\hline \multicolumn{4}{|l|}{ Lipid profile } \\
\hline HDL-cholesterol (mmol/l) & $1.43 \pm 0.38$ & $1.59 \pm 0.33$ & 0.02 \\
\hline Triglycerides $(\mathrm{mmol} / \mathrm{l})$ & $0.80(0.67 ; 0.96)$ & $1.00(0.92 ; 1.11)$ & 0.02 \\
\hline Fasting glucose (mmol/l) & $4.84(4.65 ; 5.05)$ & $5.06(4.91 ; 5.22)$ & 0.13 \\
\hline Fasting insulin $(\mu \mathrm{U} / \mathrm{ml})$ & $6.64(5.37 ; 8.22)$ & $6.46(5.86 ; 7.13)$ & 0.78 \\
\hline HOMA & $1.48(1.20 ; 1.83)$ & $1.47(1.31 ; 1.63)$ & 0.91 \\
\hline ADMA $(\mu \mathrm{mol} / \mathrm{l})$ & $0.53 \pm 0.12$ & $0.64 \pm 0.15$ & $<0.001$ \\
\hline SDMA $(\mu \mathrm{mol} / \mathrm{l})$ & $0.52 \pm 0.09$ & $0.58 \pm 0.12$ & 0.005 \\
\hline
\end{tabular}

$n$, Number of participants; HDL-cholesterol, high-density lipoprotein cholesterol; HOMA, homeostasis model assessment; ADMA, asymmetric dimethylarginine; SDMA, symmetric dimethylarginine. 


\section{Results}

\section{Characteristics of participants}

Table 2 portrays the basic characteristics of the African and Caucasian men and women. The Caucasian men were significantly taller and heavier with higher waist circumferences. They also showed higher fasting glucose, but lower $\gamma$-glutamyltransferase levels. We observed no differences in ADMA and SDMA concentrations between the African and Caucasian men, even though the African men had significantly higher diastolic BP.

When comparing the African and Caucasian women, there were no differences in age, BMI, and waist circumference between the two groups. The African women had higher $\gamma$-glutamyltransferase levels. It is striking that the Caucasian women had significantly higher ADMA and SDMA concentrations, even though the African women had higher systolic and diastolic BP.

\section{Correlations with ADMA and SDMA}

Before we explored the relationships of ADMA and SDMA with vascular function and IR, we initially performed single linear regression analyses of ADMA and SDMA with previously reported confounders in each of our four subgroups, namely age, BMI, waist circumference, estimated creatinine clearance, and $\gamma$-glutamyltransferase. The following significant correlations $(P<0.05)$ were found: ADMA and SDMA correlated with age only in the male groups $(r=0.38$ and $r=0.48$ respectively in the African men, and $r=0.43$ for ADMA in the Caucasian men). $\gamma$-glutamyltransferase also correlated only in the male groups with ADMA and SDMA $(r=0.48$ and $r=0.36$ respectively in the African men, and $r=-0.29$ for SDMA in the Caucasian men). Estimated creatinine clearance correlated significantly with ADMA only in the Caucasian men $(r=-0.30)$.

To address our aim, we then explored the correlations of ADMA and SDMA with variables associated with vascular dysfunction and IR while adjusting for confounders (Table 1). In African men, the most significant correlation was shown between PWV and ADMA $(r=0.47 ; P=0.021)$, and it was not evident in the Caucasian men. A graphical comparison of this correlation is shown in Fig. 1 (without adjustments). A significant correlation between PWV and ADMA was also shown by Caucasian women, but it was much weaker than the correlation in the African men $(r=0.26$; $P=0.008)$ and the sample size was much larger.

Significant correlations of SDMA were indicated only in the Caucasian groups (Table 1). Caucasian women showed a positive correlation between PWV and SDMA $(r=0.22 ; P=0.032)$, similar to the correlation between PWV and ADMA. Caucasian men showed no significant
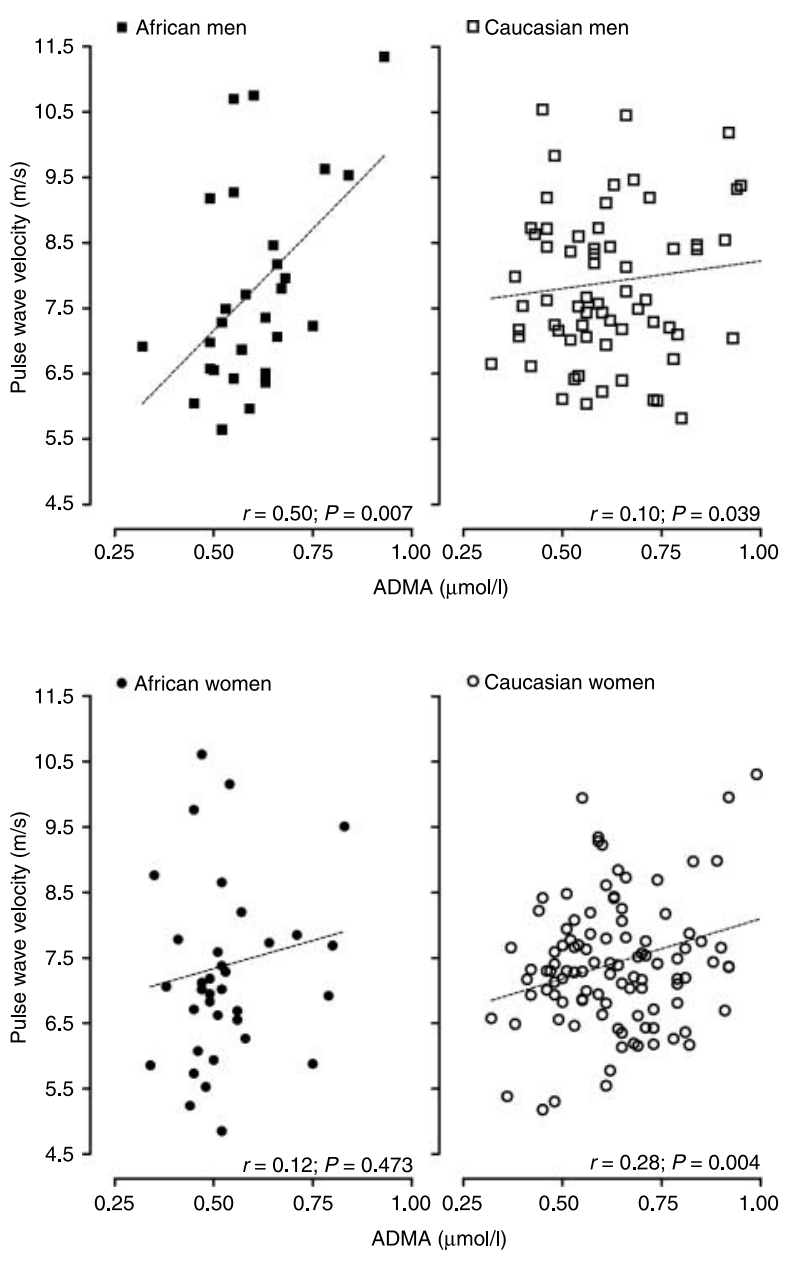

Figure 1 Unadjusted correlations between asymmetric dimethylarginine and pulse wave velocity.

correlations for ADMA, but with regards to SDMA, they indicated strong negative correlations with fasting insulin and HOMA $(r=-0.41 ; P=0.002$ in both instances), which were not observed in any of the other groups. When viewing HOMA by quartiles of SDMA (Fig. 2), the trend in Caucasian men of HOMA to decrease with an increase in SDMA was significant $(P=0.006)$. Although the HOMA trend line for Caucasian women also seem to decrease with increasing SDMA quartiles, it was not significant $(P=0.37)$.

\section{Multiple regression analyses}

With either ADMA or SDMA as dependent variable, we performed forward stepwise regression analyses in each ethnic and gender group. As outlined in Table 3, PWV entered all models with regards to ADMA (except for African women), and also for SDMA in the two female groups. PWV explained $22 \%$ of the variance in ADMA in the African male group. HOMA (log) was included as significant contributor only in the Caucasian men and 

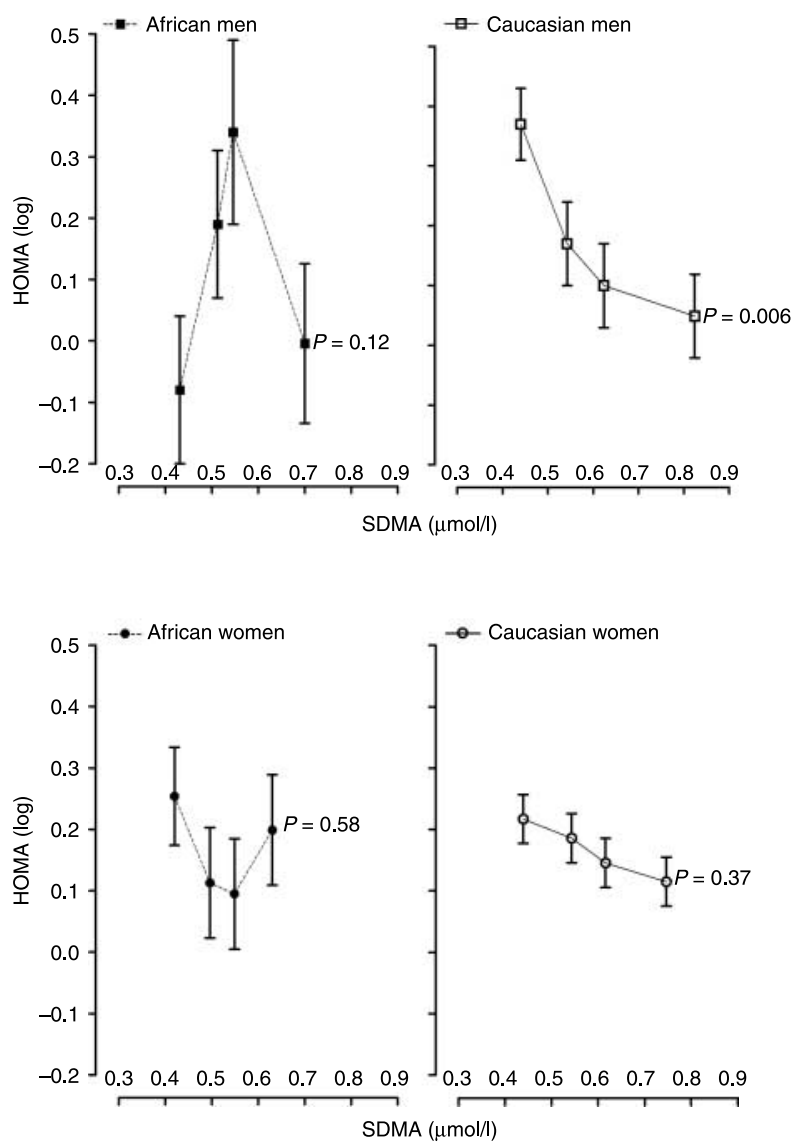

Figure 2 HOMA (log) by quartiles of plasma symmetric dimethylarginine in African and Caucasian men and women. Plotted values are least-squares means (S.E.M.) adjusted for age, body mass index, waist circumference, $\gamma$-glutamyltransferase, and estimated creatinine clearance. $P$ value denotes significance for trend.

women, being highly significant in the Caucasian men. We also repeated these analyses (not shown in Table 3) and included ethnicity in the list of independent variables. With ADMA as dependent variable, we found $R^{2}=0.165$ in men, and the model only included age $(\beta=0.44 ; P<0.001)$ and TG $(\beta=0.21 ; P=0.28)$. In women, $R^{2}=0.13$, and ethnicity was the strongest predictor $(\beta=0.31 ; P<0.001)$ and also PWV $(\beta=0.21$; $P=0.009)$. When we repeated these analyses with SDMA as dependent variable, ethnicity was a significant predictor for both men and women. For men, $R^{2}=0.20$ (HOMA: $\beta=-0.36, \quad P<0.001 ; \quad$ age: $\beta=0.29$; $P=0.004$; ethnicity: $\beta=0.21 ; P=0.04$ ) and for women, $R^{2}=0.10$ (ethnicity: $\beta=0.23, P=0.007$; PWV: $\beta=0.20 ; P=0.02$; HOMA: $\beta=-0.15 ; P=0.07$ ).

\section{Discussion}

We explored the associations of the two dimethylarginines, ADMA and SDMA, with measures of vascular dysfunction and IR in nondiabetic, nonobese, and nonsmoking African and Caucasian people from South Africa. The first key finding was a strong significant correlation of ADMA with PWV, especially evident in the African men and to a lesser extent in the Caucasian women. In African men, there seems to be a potential role for ADMA in regulating arterial stiffness, whereas this is not necessarily true for Caucasians based on the weak associations in the multivariate analyses. In multivariate analyses, PWV also explained the variance in ADMA of other groups, indicating a potential role for ADMA in regulating arterial stiffness. Since ADMA is known for its inhibitory role on NO synthase (9) and has previously been associated with cardiovascular disease $(8,10,12)$, this finding is not surprising. However, not much has been published with regards to ADMA and arterial stiffness, and the results from Weber et al. (29) revealed a relatively weak correlation between ADMA and PWV $(r=0.19$; $P=0.02)$ in a European population, which disappeared after correction for age and BP. In our study, ADMA also did not correlate with arterial stiffness in the Caucasian men, but the Caucasian women showed a weak significant positive correlation.

The strong relationship between ADMA and arterial stiffness observed in the African men could point to a different vascular mechanism in the development of cardiovascular disease previously seen in black African men $(1,7)$. It is well known that compared to Caucasians, Africans disclose larger increases in aortic and peripheral PWV after smoking (30), they show no changes in the peripheral arterial response to exercise compared to Caucasians who show significant reductions (31), and their peripheral muscular arteries show increased PWV with diabetes, with no change in Europeans (32). The next step would therefore be to investigate whether ADMA might have an important role in this regard. Our results also indicate that the concentration of ADMA in African men is not necessarily of utmost importance, since it was not different from their Caucasian counterparts. This result is in contrast to those of Melikian et al. (7) who found significantly elevated ADMA levels in African men who were matched with Caucasian men for conventional risk factors for coronary heart disease. Although our study excluded many people with increased cardiovascular risk (e.g. obesity, smoking and diabetes), we were not able to exclude hypertensive individuals, since hypertension has a high prevalence in the black South African population (6). Nevertheless, their ADMA levels were similar to the Caucasian men, even though they had significantly higher diastolic BP. It is not clear why our results are different from that of Melikian et al. (7) but it is possibly due to a different genetic and environmental background (1).

The second key finding of this study was a significant negative association between SDMA and IR only in the Caucasian men and women, being more pronounced in the men. Although SDMA was long thought to be inert 
Table 3 Forward stepwise multiple regression analyses with either asymmetric dimethylarginine (ADMA) or symmetric dimethylarginine (SDMA) as dependent variable.

\begin{tabular}{|c|c|c|c|c|}
\hline \multirow[b]{2}{*}{$R^{2}$} & \multicolumn{4}{|c|}{ African men } \\
\hline & $\begin{array}{l}\text { ADMA }(\mu \mathrm{mol} /)^{\mathrm{a}} \\
0.22 \\
\beta \text { (S.E.M.) }\end{array}$ & $P$ & $\begin{array}{l}\text { SDMA }(\mu \mathrm{mol} / /)^{b} \\
0.19 \\
\beta \text { (S.E.M.) }\end{array}$ & $P$ \\
\hline Age & - & & $0.37(0.18)$ & 0.05 \\
\hline$\gamma$-glutamyltransferase (log) & - & & $0.23(0.18)$ & 0.22 \\
\hline Pulse wave velocity & $0.50(0.17)$ & 0.006 & - & \\
\hline
\end{tabular}

\begin{tabular}{|c|c|c|c|c|}
\hline \multirow[b]{2}{*}{$R^{2}$} & \multicolumn{4}{|c|}{ Caucasian men } \\
\hline & $\begin{array}{l}0.19 \\
\beta \text { (S.E.M.) }\end{array}$ & $P$ & $\begin{array}{l}0.28 \\
\beta \text { (S.E.M.) }\end{array}$ & $P$ \\
\hline Age & $0.52(0.13)$ & $<0.001$ & $0.29(0.11)$ & 0.01 \\
\hline$\gamma$-glutamyltransferase (log) & $-0.16(0.11)$ & 0.17 & $-0.16(0.12)$ & 0.20 \\
\hline Pulse wave velocity & $-0.17(0.13)$ & 0.21 & - & \\
\hline HOMA (log) & - & & $-0.43(0.12)$ & $<0.001$ \\
\hline
\end{tabular}

\begin{tabular}{lllll}
\hline & \multicolumn{3}{c}{ African women } \\
\cline { 2 - 5 } $\boldsymbol{R}^{2}$ & 0.18 & & 0.03 & \\
\hline Age & $\beta$ (s.E.M.) & $P$ & - & \\
Mean arterial pressure & $0.49(0.21)$ & 0.03 & - & \\
$\gamma$-glutamyltransferase (log) & $-0.42(0.21)$ & 0.06 & $-0.23(0.18)$ & 0.21 \\
Triglycerides (log) & $-0.28(0.19)$ & 0.15 & $-0.23(0.18)$ & 0.15 \\
Pulse wave velocity & - & $0.17(0.19)$ & & $0.26(0.18)$ \\
\hline
\end{tabular}

\begin{tabular}{|c|c|c|c|c|}
\hline \multirow[b]{2}{*}{$R^{2}$} & \multicolumn{4}{|c|}{ Caucasian women } \\
\hline & $\begin{array}{l}0.07 \\
\beta \text { (S.E.M.) }\end{array}$ & $P$ & $\begin{array}{l}0.08 \\
\beta \text { (S.E.M.) }\end{array}$ & $P$ \\
\hline $\begin{array}{l}\text { Pulse wave velocity } \\
\gamma \text {-glutamyltransferase (log) } \\
\text { HOMA (log) }\end{array}$ & $\begin{array}{l}0.26(0.09) \\
-0.12(0.09) \\
-\end{array}$ & $\begin{array}{l}0.007 \\
0.21\end{array}$ & $\begin{array}{l}0.26(0.10) \\
- \\
-0.21(0.10)\end{array}$ & $\begin{array}{l}0.01 \\
0.04\end{array}$ \\
\hline
\end{tabular}

HOMA, homeostasis model assessment.

alndependent variables included in model: age, body mass index, mean arterial pressure, pulse wave velocity, triglycerides, and $\gamma$-glutamyltransferase.

${ }^{b}$ Independent variables included in model: age, body mass index, mean arterial pressure, pulse wave velocity, triglycerides, $\gamma$-glutamyltransferase, estimated creatinine clearance, and HOMA.

(8), recent research suggests the opposite $(8,18,22)$. We were particularly interested to determine whether this also holds true for the African population, but did not find any significant correlation of SDMA in single or multivariate analyses. This ethnic discrepancy was also confirmed in the multiple regression analyses when ethnicity was included as an independent variable, and proved to be a significant predictor of SDMA for both genders.

Interestingly, we also found a positive association of SDMA with arterial stiffness in the Caucasian women. In addition to ADMA, SDMA might also have a smaller role in contributing to arterial stiffness. A possible mechanism is demonstrated by Schepers et al. (18) who showed very recently that SDMA stimulates the production of reactive oxygen species of monocytes, and that this pro-inflammatory effect may trigger vascular pathology.

The negative relationship found between SDMA and IR in Caucasians confirms the results of Zsuga et al. (22) who studied Caucasian people from Hungary. They proposed several mechanisms for this phenomenon including increased cellular uptake of SDMA, enhanced elimination, or that the hepatic metabolic pathways of SDMA are somehow up-regulated in IR. Unfortunately, neither their cross-sectional design nor ours provide sufficient data to explain whether decreased SDMA might somehow be causal in the development of IR, but these strong relationships most certainly warrant further investigation. Importantly, it is unclear why this relationship was absent in the African subjects and whether a genetic predisposition might be accountable. Although hypertension and type 2 diabetes are common in Africans, our results could imply that it is due to other mechanisms than the pathophysiological mechanisms of SDMA, and that they might be protected from its detrimental effects as described previously in Caucasian populations $(8,19)$.

It is also worth mentioning that contradictory findings exist with regards to the relationship between 
ADMA and IR since many have reported on this finding $(13-15,33)$, whereas others $(22,34)$, including ourselves, did not observe this relationship in Caucasian, Hispanic, or African participants. Our group has previously shown that ADMA is positively correlated with parameters of glucose metabolism in Caucasian but not African women who were individually matched for age, BMI, and socioeconomic status (33). This might indicate a genetic discrepancy regarding ADMA function. It is also important to mention that this is not the first study to reveal differences in cardiometabolic risk factors between the South African Caucasian and African population groups. High prevalences of hypertension (2) have been described in Africans compared to Caucasians, with clearly different treatment approaches (35). Africans have low levels of triacylglycerol, and high levels of high-density lipoprotein-cholesterol and fibrinogen (36) compared to Caucasians in South Africa, and several genetic differences with regards to type 2 diabetes and hypertension have also been described (2). The results of the present study therefore contribute to elucidating the different mechanisms at play in the development of hypertension and IR in these two ethnic groups.

\section{Study limitations}

The present study suffers from some of the same limitations as many other studies. One limitation of our study is the relatively small sample size. The number of participants was, however, enough to confirm statistical power and it was similar to the previously published African data on ADMA (7), but additionally included data on women. Another limitation would be the cross-sectional design of this study, which could not infer causality. Regarding the use of HOMA, although it is an accepted and validated model for the characterization of IR (37), it is still a proxy measurement. Furthermore, it is not clear whether the association between HOMA and SDMA is simply a reflection of the relationship between insulin and SDMA as was shown in Table 1. It has been previously reported that hyperinsulinemia is able to induce the expression of $y+$ transporters that serve as the primary route for cellular uptake of L-arginine, ADMA, and SDMA (22, 38 ), and thus there are direct links between SDMA and insulin. With regards to the participants, there was a difference in socioeconomic status between the African and Caucasian individuals who volunteered to participate in the study. In an attempt to make the groups comparable with regards to traditional cardiovascular risk factors, we did, however, exclude 515 participants who were either HIV infected, obese, diabetic, smoking or used antihypertensive, lipid-lowering, or anti-inflammatory medication. Therefore, we are convinced that our sub-sample is suitable to provide the first results on SDMA in Africans, as well as some novel associations with arterial stiffness in this population group.
In conclusion, we found a strong positive relationship between ADMA and arterial stiffness in African men, even though their ADMA levels were not elevated. ADMA might therefore serve as a novel-therapeutic target to improve NO bioavailability. Additionally, we have found a strong negative relationship between SDMA and IR in Caucasians, which was completely absent in the African men and women. The ethnic discrepancy is difficult to explain but might indicate different mechanisms for the development of IR in Africans.

\section{Declaration of interest}

$\mathrm{E} \mathrm{S}$ and $\mathrm{R} \mathrm{H} \mathrm{B}$ are named as inventors on patents relating to dimethylarginine assays and receive royalties from these. All other authors have declared no conflicts of interest.

\section{Funding}

We are also grateful towards our sources of support: the South African National Research Foundation (GUN 2073040), the Medical Research Council, and the Africa Unit for Transdisciplinary Health Research (AUTHeR) of the North-West University (Potchefstroom campus).

\section{Acknowledgements}

We thank the participants, as well as all supporting staff and postgraduate students, for their involvement in this project.

\section{References}

1 Opie LH \& Seedat YK. Hypertension in sub-Saharan African populations. Circulation $20051123562-3568$.

2 Seedat YK. Hypertension in black South Africans. Journal of Human Hypertension 199913 96-103.

3 Steyn K, Kazenellenbogen JM, Lombard CJ \& Bourne LT. Urbanization and the risk for chronic diseases of lifestyle in the black population of the Cape Peninsula, South Africa. Journal of Cardiovascular Risk 19974 135-142.

4 van Rooyen JM, Kruger HS, Huisman HW, Wissing MP, Margetts BM, Venter CS \& Vorster HH. An epidemiological study of hypertension and its determinants in a population in transition: the THUSA study. Journal of Human Hypertension $2000 \mathbf{1 4}$ 779-787.

5 De Courten M, McCarty D \& Zimmet P. Diagnosis: the scale of the problem and future risks. In Type 2 Diabetes, Prediction, and Prevention, pp 17-36. Ed. G Hitman, Chichester: John Wiley \& Sons, 1999.

6 Sliwa K, Wilkinson D, Hansen C, Ntyintyane L, Tibazarwa K, Becker A \& Stewart S. Spectrum of heart disease and risk factors in a black urban population in South Africa (the Heart of Soweto Study): a cohort study. Lancet 2008371 915-922.

7 Melikian N, Wheatcroft SB, Ogah OS, Murphy C, Chowienczyk PJ, Wierzbicki AS, Sanders TA, Jiang B, Duncan ER, Shah AM \& Kearney MT. Asymmetric dimethylarginine and reduced nitric oxide bioavailability in young Black African men. Hypertension 200749 873-877.

8 Kiechl S, Lee T, Santer P, Thompson G, Tsimikas S, Egger G, Holt DW, Willeit J, Xu Q \& Mayr M. Asymmetric and symmetric dimethylarginines are of similar predictive value for cardiovascular risk in the general population. Atherosclerosis 2009205 261-265.

9 Boger RH. Asymmetric dimethylarginine (ADMA) and cardiovascular disease: insights from prospective clinical trials. Vascular Medicine 200510 S19-S25. 
10 Boger RH, Sullivan LM, Schwedhelm E, Wang TJ, Maas R, Benjamin EJ, Schulze F, Xanthakis V, Benndorf RA \& Vasan RS. Plasma asymmetric dimethylarginine and incidence of cardiovascular disease and death in the community. Circulation 2009119 1592-1600.

11 Schulze F, Maas R, Freese R, Schwedhelm E, Silberhorn E \& Boger RH. Determination of a reference value for $N(G), N(G)$ dimethyl-L-arginine in 500 subjects. European Journal of Clinical Investigation $200535622-626$.

12 Zoccali C. Asymmetric dimethylarginine (ADMA): a cardiovascular and renal risk factor on the move. Journal of Hypertension 200624 611-619.

13 Stuhlinger MC, Abbasi F, Chu JW, Lamendola C, McLaughlin TL, Cooke JP, Reaven GM \& Tsao PS. Relationship between insulin resistance and an endogenous nitric oxide synthase inhibitor. Journal of the American Medical Association 2002287 1420-1426.

14 McLaughlin T, Stuhlinger M, Lamendola C, Abbasi F, Bialek J, Reaven GM \& Tsao PS. Plasma asymmetric dimethylarginine concentrations are elevated in obese insulin-resistant women and fall with weight loss. Journal of Clinical Endocrinology and Metabolism 200691 1896-1900.

15 Sydow K, Mondon CE \& Cooke JP. Insulin resistance: potential role of the endogenous nitric oxide synthase inhibitor ADMA. Vascular Medicine 200510 S35-S43.

16 Vallance P. Importance of asymmetrical dimethylarginine in cardiovascular risk. Lancet 2001358 2096-2097.

17 Vallance $\mathrm{P} \&$ Leiper J. Cardiovascular biology of the asymmetric dimethylarginine:dimethylarginine dimethylaminohydrolase pathway. Arteriosclerosis, Thrombosis, and Vascular Biology 200424 1023-1030.

18 Schepers E, Glorieux G, Dhondt A, Leybaert L \& Vanholder R. Role of symmetric dimethylarginine in vascular damage by increasing ROS via store-operated calcium influx in monocytes. Nephrology, Dialysis, Transplantation 200924 1429-1435.

19 Schulze F, Carter AM, Schwedhelm E, Ajjan R, Maas R, Von Holten RA, Atzler D, Grant PJ \& Boger RH. Symmetric dimethylarginine predicts all-cause mortality following ischemic stroke. Atherosclerosis, 2009 DOI:10.1016/j.atherosclerosis.2009. 06.039 .

20 Closs EI, Basha FZ, Habermeier A \& Forstermann U. Interference of L-arginine analogues with L-arginine transport mediated by the $\mathrm{y}+$ carrier hCAT-2B. Nitric Oxide 19971 65-73.

21 Kielstein JT, Salpeter SR, Bode-Boeger SM, Cooke JP \& Fliser D. Symmetric dimethylarginine (SDMA) as endogenous marker of renal function - a meta-analysis. Nephrology, Dialysis, Transplantation $2006212446-2451$.

22 Zsuga J, Torok J, Magyar MT, Valikovics A, Gesztelyi R, Lenkei A, Csiba L, Keki S, Zsuga M \& Bereczki D. Dimethylarginines at the crossroad of insulin resistance and atherosclerosis. Metabolism 200756 394-399.

23 Cockroft DW \& Gault MH. Prediction of creatinine clearance from serum creatinine. Nephron $19761631-41$.

24 Schwedhelm E, Tan-Andresen J, Maas R, Riederer U, Schulze F \& Boger RH. Liquid chromatography-tandem mass spectrometry method for the analysis of asymmetric dimethylarginine in human plasma. Clinical Chemistry 200551 1268-1271.

25 Schwedhelm E, Maas R, Tan-Andresen J, Schulze F, Riederer U \& Boger RH. High-throughput liquid chromatographic-tandem mass spectrometric determination of arginine and dimethylated arginine derivatives in human and mouse plasma. Journal of Chromatography. B, Analytical Technologies in the Biomedical and Life Sciences $2007851211-219$.

26 Norton K \& Olds T. Anthropometrica. A Textbook of Body Measurement for Sports and Health Courses Sydney: University of New South Wales Press, 1996.

27 Paiva H, Lehtimaki T, Laakso J, Ruokonen I, Tervonen R, Metso S, Nikkila M, Wuolijoki E \& Laaksonen R. Dietary composition as a determinant of plasma asymmetric dimethylarginine in subjects with mild hypercholesterolemia. Metabolism 200453 1072-1075.

28 Conigrave KM, Davies P, Haber P \& Whitfield JB. Traditional markers of excessive alcohol use. Addiction 200398 31-43.

29 Weber T, Maas R, Auer J, Lamm G, Lassnig E, Rammer M, O'Rourke MF, Boger RH \& Eber B. Arterial wave reflections and determinants of endothelial function a hypothesis based on peripheral mode of action. American Journal of Hypertension 2007 20 256-262.

30 Lemogoum D, van Bortel LM, Leeman M, Degaute JP \& van de Borne P. Ethnic differences in arterial stiffness and wave reflections after cigarette smoking. Journal of Hypertension $2006 \mathbf{2 4}$ 683-689.

31 Heffernan KS, Jae SY \& Fernhall B. Racial differences in arterial stiffness after exercise in young men. American Journal of Hypertension $2007 \mathbf{2 0} 840-845$.

32 Strain WD, Chaturvedi N, Dockery F, Shiff R, Shore AC, Bulpitt CJ \& Rajkumar C. Increased arterial stiffness in Europeans and African Caribbeans with type 2 diabetes cannot be accounted for by conventional cardiovascular risk factors. American Journal of Hypertension 200619 889-896.

33 Reimann M, Schutte AE, Malan NT, Schwarz PE, Benndorf RA, Schulze F \& Boger RH. Asymmetric dimethylarginine is associated with parameters of glucose metabolism in Caucasian but not in African women from South Africa. Experimental and Clinical Endocrinology $\mathcal{E}$ Diabetes 2007115 600-605.

34 Garcia RG, Perez M, Maas R, Schwedhelm E, Boger RH \& Lopez-Jaramillo P. Plasma concentrations of asymmetric dimethylarginine (ADMA) in metabolic syndrome. International Journal of Cardiology 2007122 176-178.

35 Brewster LM, van Montfrans GA \& Kleijnen J. Systematic review: antihypertensive drug therapy in black patients. Annals of Internal Medicine $2004141614-627$.

36 Vorster HH, Jerling JC, Steyn K, Badenhorst CJ, Slazus W, Venter CS, Jooste PL \& Bourne LT. Plasma fibrinogen of black South Africans: the BRISK study. Public Health Nutrition 19981 169-176.

37 Wallace TM, Levy JC \& Matthews DR. Use and abuse of HOMA modeling. Diabetes Care 200427 1487-1495.

38 Simmons WW, Closs EI, Cunningham JM, Smith TW \& Kelly RA. Cytokines and insulin induce cationic amino acid transporter (CAT) expression in cardiac myocytes. Regulation of L-arginine transport and no production by CAT-1, CAT-2A, and CAT-2B. Journal of Biological Chemistry 1996271 11694-11702.

Received 2 November 2009

Accepted 7 December 2009 